\title{
Insect-Computer Hybrid Robot
}

\author{
Yao Li and Hirotaka Sato ${ }^{1}$
}

\begin{abstract}
An insect-computer hybrid robot, often referred to as a biological machine or an insect cyborg, is the fusion of a living insect platform and artificial microdevices, including stimulators, sensors, and computers. When compared with the artificial robots, a hybrid robot can be operated as an autonomous mobile machine with low energy consumption and hardware costs. A hybrid machine can verify various biological hypotheses, such as function determination, by stimulating a muscle or any other structure.
\end{abstract}

Keywords: Animal Hybrid Robot; Electrical Stimulation; Wireless Devices; Insect Flight; Micro Drone; Biobot.

\section{INTRODUCTION}

Insects are the largest class among all animals due to their large diversity. They range in size from less than 1 millimeter to more than 20 centimeters. Their lengthy evolution has produced several modes of locomotion such as flying, walking, swimming, jumping, and digging. These efficient and sophisticated functions of insects allow them to spread extensively throughout the world. Insect biomimetics has inspired several engineers and scientists for centuries ${ }^{1,2}$.

Because of the advances in electronics and control technology, artificial biomimetic robots have recently emerged. Several artificial-legged robots have been demonstrated with flexible steps, which were inspired by the walking gaits of insects ${ }^{3-5}$. With the recent developments in micromanufacturing, an insect-scale flapping-wing micro air vehicle, the RoboBee, was launched by Ma, Chirarattananon ${ }^{6}$. It weighed $80 \mathrm{mg}$ and exhibited a 3-cm wingspan. However, building elaborate robots that can move like insects is not simple. Even state-of-the-art artificial robots are far behind their natural exemplars in terms of stability, agility, and maneuverability due to our lack of knowledge about insect locomotion and due to the limitations of current control technology. A natural bee can easily hover in almost all the outdoor conditions, whereas a RoboBee can barely maintain stability in mild winds due to the lack of an analytical model ${ }^{7}$. Furthermore, the lifetime of a RoboBee is less than 10 minutes due to the structural and material limitations ${ }^{8,9}$. A cockroach can travel quickly over uneven ground and it is self-righting, which have not yet been achieved by insect-like walking robots $^{10}$. The capacity of the power source is another practical limitation. DelFly is a wireless flapping-wing micro air vehicle, which weighs 3 grams and exhibits a wingspan of $10 \mathrm{~cm}$. Despite attempts to reduce the structural weight and to increase the payload capacity, it can fly for only up to 3 minutes due to battery limitations ${ }^{11}$.

Insect-scale robots require a precise mechanical structure and a complex control system. However, in the previous decade, some researchers have obtained a solution to these technical limitations, which is to hybridize a microcomputer with a living insect. Such an insect-computer hybrid robot, often referred to as a biological machine or an insect cyborg, is the fusion of a living insect platform and artificial electronic microdevices, which are used to either completely or partially control the insect's movement. Therefore, the microdevices embedded on the insect transmit commands to stimulate the neuromuscular sites in the insect, which trigger the desired motor actions and behaviors instead of the insect executing its own commands and generating its own

\footnotetext{
${ }^{1}$ School of Mechanical and Aerospace Engineering, Nanyang Technological University, 50 Nanyang Avenue, Singapore 639798. Correspondence should be addressed to: H.S. (hirosato@ntu.edu.sg). Published online 27 August 2018; doi:10.1142/S2529732518500025

This is an Open Access article published by World Scientific Publishing Company. It is distributed under the terms of the Creative Commons Attribution 4.0 (CC-BY) License. Further distribution of this work is permitted, provided the original work is properly cited.
} 
motion. Furthermore, an insect-computer hybrid robot could solve the limitations of the power source because its motion is generated by the insect rather than by the artificial actuators. The power consumption of electrical stimulation is very low, in the order of microwatts, as compared with the power requirements of artificial robots, which can be in the order of a few watts or more.

Insect-computer hybrid systems have been realized since the $1990 \mathrm{~s}^{12-14}$. As smaller and lower-power microcontrollers and radio systems continue to appear on the market, the performance of insect-computer hybrid robots has continually improved. Researchers have demonstrated several wireless microdevices for controlling the walking and flying of insects. Electronic backpacks mounted on cockroaches and beetles are designed to transform the insects into controllable walking robots ${ }^{15-20}$. Moreover, initiating flight and turning in flight of moths and beetles have been demonstrated using insect-mountable microdevices with wireless communications, which made the insects into flying robots ${ }^{21-28}$. We believe that insect-computer hybrid systems will be utilized in real applications since the microcontrollers are getting stronger and our knowledge of the biological systems keeps advancing.

We have continuously studied insect-computer hybrid robots since 2007. Electrically induced walking steps of a beetle have been clearly and reliably demonstrated ${ }^{18-20}$. Meanwhile, our group has proposed two methods to initiate flight by a beetle: electrical muscular stimulation (of the dorsal longitudinal muscles) and electrical neuronal stimulation (of the optic lobes; Figure 1a) ${ }^{21,29}$. We have achieved turning in flight by applying electrical signals to the flight mechanism of the beetle $21,25,26,30$.

Furthermore, using a hybrid system, we can verify biological hypotheses. Suppose there is some muscle or structure whose function is unknown and we have a hypothesis about its function in locomotion. We can test this hypothesis by demonstrating the function through stimulation of the muscle of interest.

\section{CONTROL TECHNOLOGY AND DEVICES}

Many studies on insect-computer hybrid robots have been done using cockroaches, moths, and beetles because they have been thoroughly studied by biologists and they are large enough to carry electronic devices. Controlling these hybrid robots has been accomplished by applying an exogenous stimulation, such as an electrical stimulation $^{29,31-33}$, a visual stimulation ${ }^{34}$, a thermal stimulation ${ }^{23}$, or a chemical injection ${ }^{35}$. Due to its practicality and reliability, electrical stimulation is the most commonly used method.
The electrical stimulation must be applied exactly to the muscle tissues or neurons of interest via implanted electrodes. A certain level of anatomical knowledge and surgical skill is required to ensure both the safety of the insect and the stimulation effect (Figure 1). The stability of the electrodes is another important issue. Vibrations from the insect's movements and interference from its legs or wings inevitably cause the electrodes to drift over time. Moreover, the shock from a crash or hard impact may lead to mechanical failures of the electrodes ${ }^{21}$. Thus, implanted electrodes are typically sealed or stuck onto the exoskeleton of the insect ${ }^{25,26,30}$. In our experiments, our group used thin silver wires as stimulation electrodes, which reached the targets through small holes bored in the insects' cuticles. Melted beeswax was dripped over the holes to cover them, as beeswax quickly solidifies to immobilize the wires.

From the beginning, tethering has often been adopted to study insect locomotion. The study insect is fixed to a holder or loosely wired in a limited area for the recording or stimulation. However, tethered experiments restrict the movement of the insects. Consequently, the behavior of tethered insects may be different from their natural behavior ${ }^{36}$. The development of electronics has inspired an alternative approach: the mounting of wireless microdevices on insects. Early insectmountable devices were simply used to collect and transmit electromyograms from flying locusts and moths ${ }^{12,14,37,38}$. The lack of on-board digital processing, programmability, and efficient radio communications made insect control with them impossible ${ }^{2}$. Insect-mountable devices with microcontrollers and efficient transceivers have been demonstrated in the last decade. Specifically, Hinterwirth, Medina ${ }^{24}$ developed a miniature stimulator board based on a PIC16F688 microcontroller with a radio-frequency transmitter. The board was attached to the ventral side of moths to control their flight speed. $\mathrm{Li}, \mathrm{CaO}^{26}$ used an electrical backpack with an IEEE 802.15.4 compliant microcontroller to transmit data and stimulate flying beetles. A CC2531 microcontroller board, which implemented the Zigbee communication protocol, was used to control the walking of a cockroach ${ }^{16}$. Overall, wireless insect-mountable devices enabled by new electronic technology provide a powerful experimental tool that will likely see practicable application in insect-computer hybrid robots.

Effective motion-tracking systems are necessary for studying insect locomotion control. The evaluation of control outcomes is based on recordings from motion-tracking systems. Displacement sensors, which are widely used in tethered experiments ${ }^{39,40}$, are not suitable for free-moving insects due to their small effective range and limited multi-object identification. The method most commonly used for tracking moving insects is image processing of video recordings $\mathrm{s}^{28,41,42}$. The moving trajectory of an insect can 

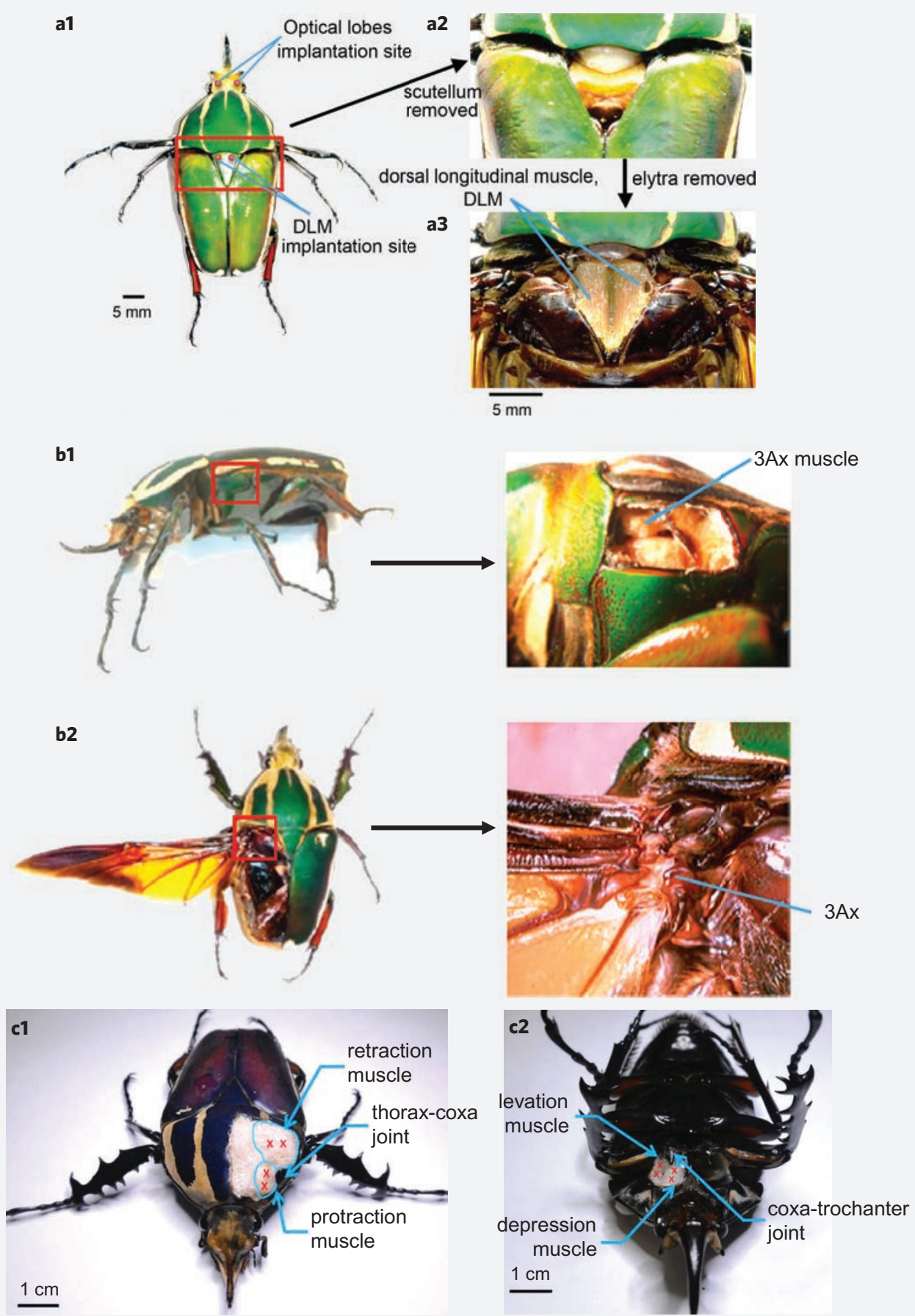

Figure 1. Anatomical views of a beetle and the implantation sites for electrical stimulation. (a1) Dorsal view of a beetle with the implantation sites for neuronal stimulation of the optic lobes and muscular stimulation of the dorsal longitudinal muscles (DLM) marked. Electrodes were inserted through small holes bored in the scutellum into muscle tissue and in the head into optical lobes. Close-up views of the dorsal thorax (a2) after removal of the scutellum and (a3) after exposing the dorsal longitudinal muscles. Side view (b1) and dorsal view (b2) of a beetle. The insets are close-up views of the red squares showing the third axillary ( $3 \mathrm{Ax}$ ) muscle (upper) and $3 \mathrm{Ax}$ sclerite (lower). (c1) The protraction and retraction muscles enable the leg to move forwards and backwards about the thorax-coxa joint. (c2) The levator and depressor muscles enable elevation and depression of the leg about the coxa-trochanter joint. Red crosses denote the implantation sites for the stimulated muscles. 
a1

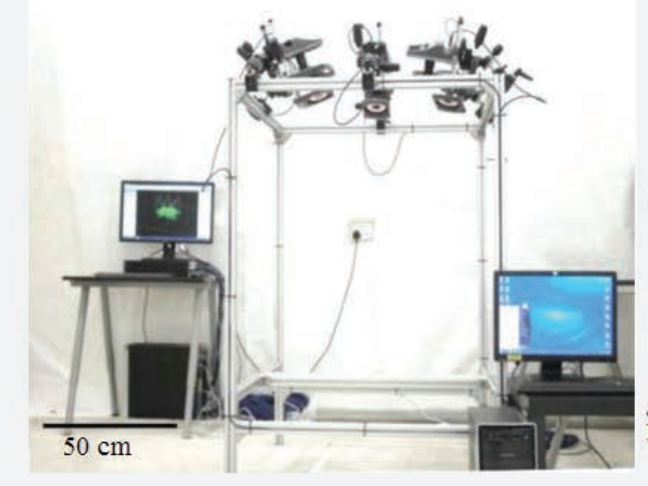

a2

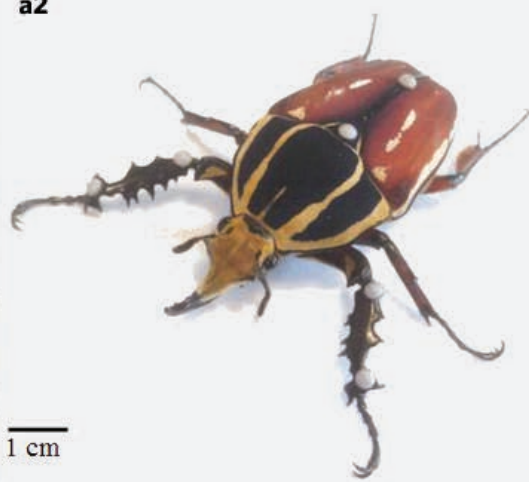

b1

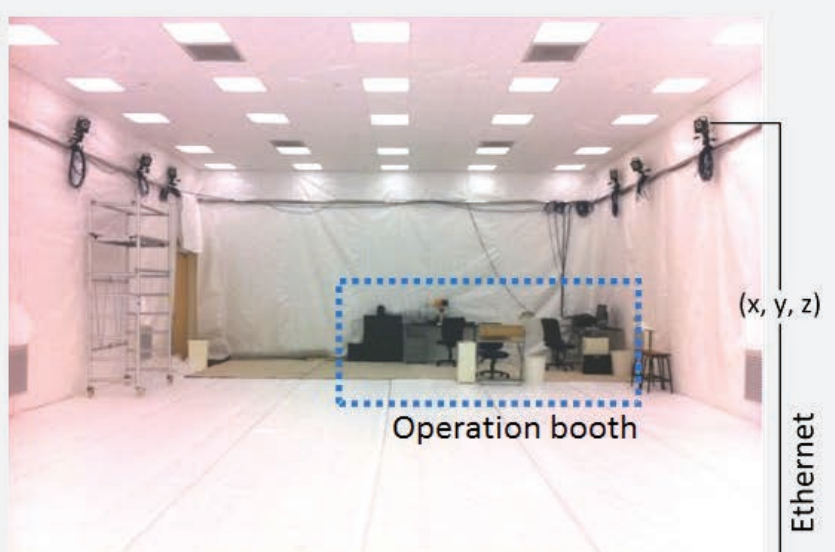

b2

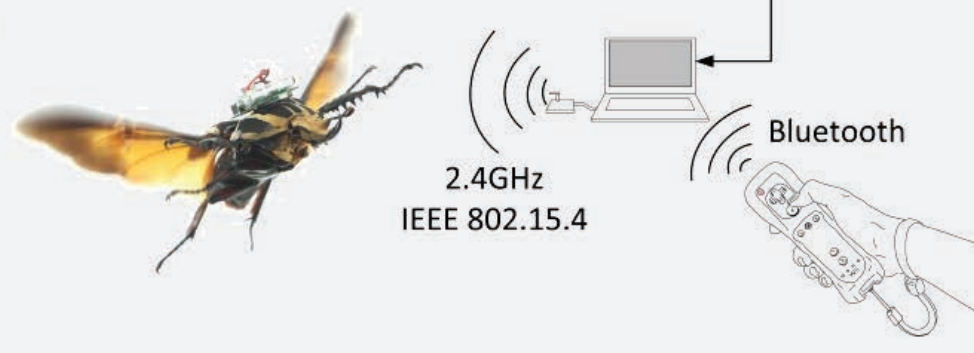

Figure 2. Motion capture system for studying insect locomotion. (a1) A motion capture system with six VICON cameras to investigate a beetle's walking gait investigation. The system can reconstruct and export the three-dimensional coordinates of retro-reflective markers. (a2) Six retro-reflective markers were attached onto a beetle, two markers on each foreleg to capture the movement of the tibia segments and two additional markers on the back to capture the body orientation. (b1) A motion capture system with 18 VICON cameras was equipped in a flight capture room $(9 \mathrm{~m} \times 16 \mathrm{~m} \times 4 \mathrm{~m})$. (b2) A beetle with a tiny backpack could fly freely around the room. Commanded by a Wii remote controller, the backpack applies an electrical stimulation to the beetle. The battery of the backpack was wrapped in retro-reflective tape, which enabled three-dimensional trajectory tracking of the flying beetle.

be extracted and analyzed in software frame by frame. Another practical method is an infra-red motion capture system, which is sensitive to retro-reflective markers ${ }^{35,43}$. Using multiple infra-red cameras that can detect a retro-reflective material, the positions of retro-reflective markers can be resolved by a computing server. Thus, it is possible to record a moving insect by simply attaching markers to it. Our group has implemented infra-red-camera-based 3D motion capture systems for tracking the small and large movements of an insect. One system enclosing a small volume has a high resolution of the order of a micrometer. It has been employed to track the detailed movement of leg swings and wingbeats (Figure 2a). Another system in a large room (9 m width $x$ $16 \mathrm{~m}$ length $\times 4 \mathrm{~m}$ height) collects the path of a flying insect (Figure 2b). However, due to its relatively low resolution of up to $1 \mathrm{~cm}$, the latter cannot track the orientation of the flying 
a1

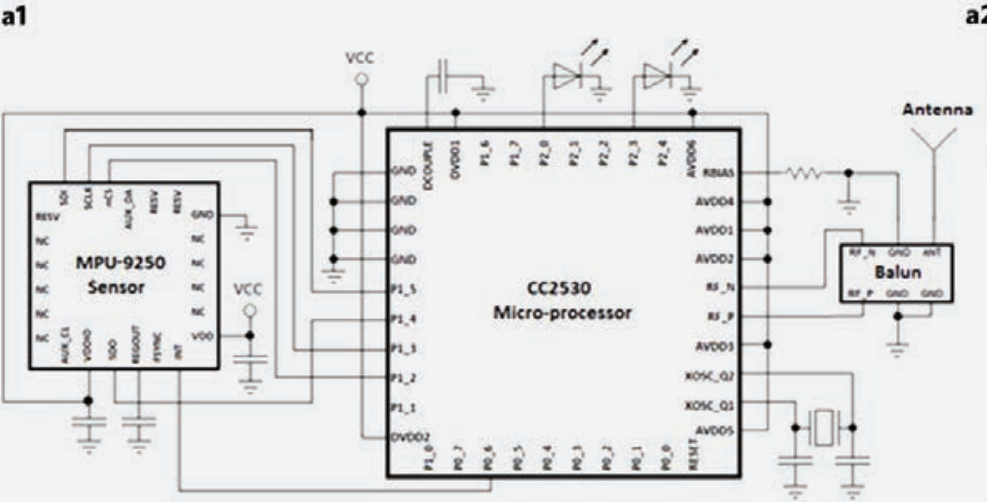

a2

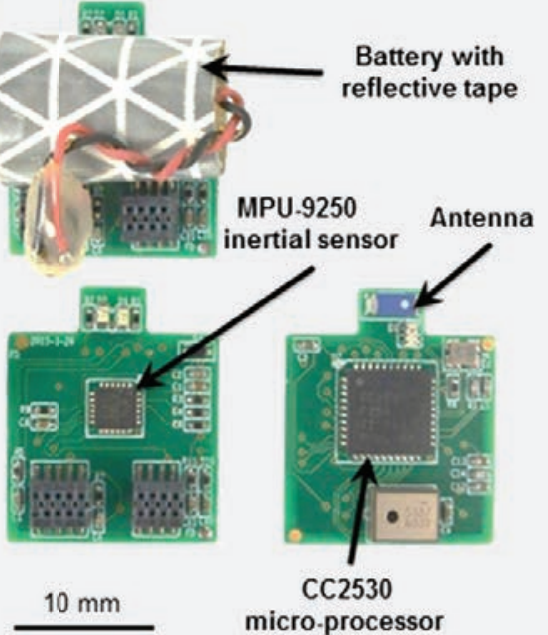

b

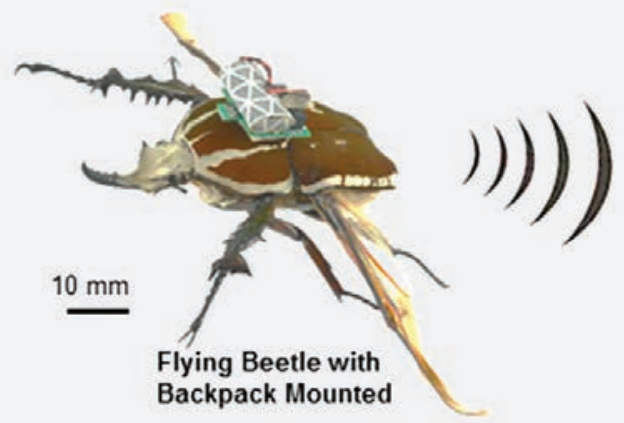

$2.4 \mathrm{GHz}$
IEEE 802.15 .4

Computer with

Custom Software

Figure 3. An inertia sensor implemented in a backpack. Schematic design (a1) and finished fabrication (a2) of a wireless backpack with an inertia sensor (dimensions: $15 \mathrm{~mm} \times 15 \mathrm{~mm}$; masses: printed circuit board assembly = $640 \mathrm{mg}$, assembly with battery = $1310 \mathrm{mg}$ ). (b) Attached to a beetle, the backpack could communicate wirelessly with a computer via a transceiver station (IEEE 802.15.4 protocol). Data from the inertia sensor were processed and transmitted to the computer at a sampling rate of $100 \mathrm{~Hz}$.

insect. We integrated an inertia measurement unit to measure the angular velocity and the acceleration of the flying insect (Figure 3) ${ }^{44}$.

\section{WALKING CONTROL}

Cockroaches are the mostly widely used insect platform for walking hybrid robots. The first walking hybrid robot was demonstrated on a cockroach by Holzer, Shimoyama ${ }^{13}$, who controlled the cockroach's walking direction by stimulating its antenna. A custom printed circuit board with wireless communication and a programmable microcontroller was recently used as the backpack to generate stimulation signals for a cockroach ${ }^{15-17,42}$. Yang, Chun ${ }^{32}$ stimulated the leg ganglia of a spider to realize left and right steering control. An Arduino Uno board was utilized to generate the electrical stimulation signals. Involuntary spasms in the leg of a spider were induced by electrical stimulation to steer the spider in the contralateral direction. Moreover, a tiny wireless stimulator was designed to control the walking of a darkling beetle (Figure $\mathbf{4 a})^{19}$. Turning and backward walking were controllable via unilateral antenna stimulation and alternating stimulation of the left and right antennae, respectively. The ultralightweight living robot was able to perform graded responses and backward walking. These are necessary for the precise motion control and efficient motion planning required by an autonomous hybrid robot. All the stimulations above were implemented via neuronal stimulation, which is the most common method for the locomotion control of insect-computer hybrid robots.

Apart from neuronal stimulation, a more straightforward method, muscular stimulation, has been applied on insect-computer hybrid robots. In preliminary studies by Cao, Zhang ${ }^{43}$ and Zhang, $\mathrm{CaO}^{45}$, the muscles of a beetle's foreleg were graded and closed-loop controlled by electrical pulses. The foreleg was transformed into a micro-actuator with high 

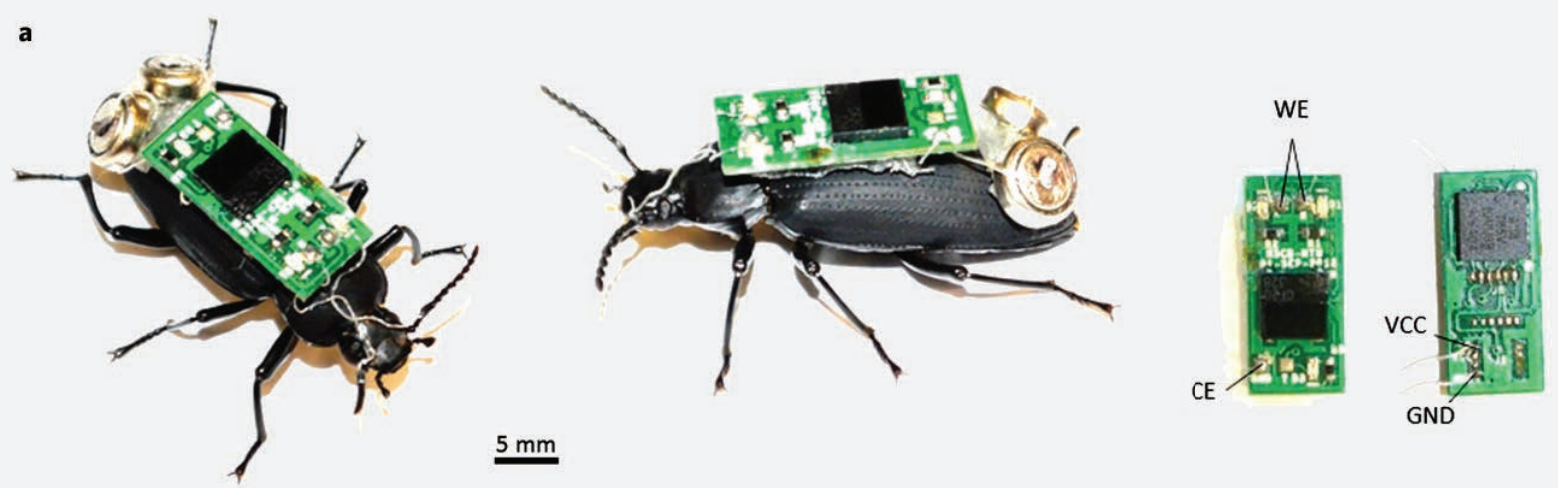

b



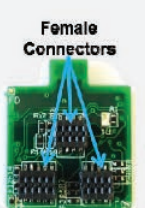

Top

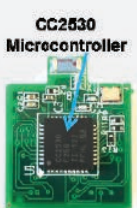

Bottom

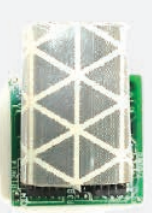

Top + Battery

Figure 4. Two insect-computer hybrid walking robots and their backpacks. (a) A wireless stimulator backpack mounted on a living insect platform (Zophobas morio), with two working electrodes (WE), one implanted in each antenna, and one counter electrode (CE) implanted in the pronotum of the beetle. (b) Another insect-computer hybrid walking robot fully assembled on a living insect platform (Mecynorrhina torquata). The CC2530 microcontroller was programmed to elicit leg motion by stimulating eight leg muscles sequentially via 16 wire electrodes.

repeatability and low power consumption. The muscular stimulation of the beetle's leg created new possibilities for controlling insect walking by permitting the modulation of the magnitude and speed of individual leg movements. Furthermore, the precise control of the insect leg enabled the development of an insect-computer hybrid legged robot. Through controlling the protraction/retraction and elevation/ depression muscle pairs of both forelegs sequentially (Figure 1c), Cao, Zhang ${ }^{18}$ commanded the beetle to walk using either the tripod or galloping walking gait, which were developed by studying the natural walking gait of the beetle. The step length and walking speed were both adjustable by altering the sequence and duration of muscle stimulation. This was the first demonstration of an insect-computer hybrid robot with user-adjustable walking gait, step length, and walking speed. Moreover, a wireless control backpack was developed for the beetle and this enabled the insect-computer hybrid system to be remotely controlled (Figure $\mathbf{4 b})^{20}$.

\section{FLIGHT INITIATION}

Compared to walking, flying is much faster and more suitable for long distances. The first step is to initiate flight initiation, which is quite important role in flight control. Several methods for stimulating flight initiation have been proposed for insects. Specifically, thermal stimulation via heat coils at the antennal base of beetle induced flight by increasing the temperature of the antennae to $43^{\circ} \mathrm{C}^{23}$. We believe that quickly heating the antennal base is harmful to the beetle, as it is close to many sensory organs. Electrical stimulation of the optic lobe of a beetle, which is between the brain and the compound eyes, was shown to start and stop wing oscillations by using $100 \mathrm{~Hz}$ 
pulses and a single long pulse, respectively ${ }^{21}$. Similarly, Bozkurt, Gilmour $\mathrm{Jr}^{22}$ demonstrated flight initiation of moths by applying electrical pulses to the antennal lobe. In addition to neuronal stimulation, Choo, $\mathrm{Li}^{29}$ proposed electrical muscular stimulation of the dorsal longitudinal muscles of a beetle to unfold and oscillate the wings.

Our group investigated the reliability and safety of neuronal stimulation and muscular stimulation for flight initiation. Neuronal stimulation was applied near the optic lobes of a beetle. These are part of the protocerebrum and extend from the brain to the retina of the visual receptors (Figure 1a) ${ }^{2}$. It was found that stimulation of the optic lobes can change photoreception in the brain and thus, modulate flight initiation $^{21,46}$. Following previous studies, Choo, $\mathrm{Li}^{29}$ applied $100 \mathrm{~Hz}$ pulse trains to the optic lobes to initiate flight by a beetle. The stimulation of the optic lobes induced wing oscillations in all beetles tested. However, after 10 consecutive stimulations, the beetles became unsteerable in the air and they could not sustain their flight for even $10 \mathrm{~s}$. The same reaction (unfolding and oscillating the wings but losing flight control) was observed in blindfolded beetles whose compound eyes were covered by beeswax and plasticine. After removing the cover, all beetles regained their normal flight ability. The results led to the conclusion that stimulation of the optic lobe damaged the photoreception of the beetles. Meanwhile, muscular stimulation, recently proposed by Choo, $\mathrm{Li}^{29}$, was directly applied to the dorsal longitudinal muscles of a beetle, which are a pair of indirect flight muscles in the thorax (Figure 1a). The dorsal longitudinal muscles generate wing oscillations. Contraction of the muscle pair depresses the wings. The implantation of electrodes caused negligible damage to the muscle tissues and did not result in the loss of flight ability. The muscular stimulation induced wing oscillations with an average success rate of $91 \%$, which was almost as high as that for optic lobe stimulation. The muscular stimulation had a short response time $(0.77 \mathrm{~s})$, close to that for optic lobe stimulation $(0.5 \mathrm{~s})$ and short enough for practical applications. Notably, none of the beetles tested lost their flight ability after 10 trials, confirming that no crucial damage was caused to the flight system.

\section{FLIGHT DIRECTIONAL CONTROL}

\section{Wings}

Insects steer in air mainly by changing the shape of their flexible wings as they rotate around the wing hing $\mathrm{e}^{47,48}$. The aerodynamic forces generated by the wings are modulated by the wingbeat frequency, stroke plane angle, and stroke amplitude ${ }^{47}$. The flight muscles can be electrically stimulated to control the wingbeats.

For on-demand wing actuation and flight direction control, Bozkurt, Gilmour ${ }^{33}$ designed a four-channel microprobe to stimulate the dorsal longitudinal muscles and the dorso-ventral muscles of a loosely tethered moth. When a unilateral stimulation pulse was applied to a forward flying moth, a backward shift of the wing occurred on the stimulated side. Thus, a yaw turn towards the stimulated side was induced.

Moreover, researchers have demonstrated the wireless control of beetle flight by stimulating several flight muscles. Specifically, Sato and Berry investigated the basalar muscle in free flight ${ }^{21}$. A working electrode was implanted into both the left and right basalar muscles to apply stimulation. A common counter electrode was inserted into the posterior pronotum. The basalar muscle is a major flight muscle that reinforces the downward stroke in wing flapping. The electrical pulsing of a basalar muscle increased the power output from the stimulated side, which could induce a contralateral turn in flight.

In addition, the subalar muscle was studied by Vo Doan, $\mathrm{Li}^{30}$. This flight muscle is between the subalar sclerite and the leg coxa and is responsible for twisting the wing. Stimulation of the subalar muscle was achieved by two electrodes inserted into the muscle tissue. The effective range of the stimulus frequency was from 70 to $100 \mathrm{~Hz}$. Pitching control of a beetle was realized by stimulating the subalar muscle in free flight. The body pitch angle increased due to the contraction of the subalar muscle and the flight velocity decreased accordingly.

Another experiment revealed that a contraction of the third axillary muscle pulled the third axillary of the wing base dorsally and inward (Figure $\mathbf{1 b})^{25}$. As a consequence, the wing stroke amplitude reduced and the force generated decreased, which means an ipsilateral turn would be induced (Figure 5). However, since the 19th century, the third axillary muscle was considered to function in wing folding, rather than flight steering. With the stimulation via a wireless backpack and the analysis of wing flapping, this study redefined the function of the muscle in left-right steering. Moreover, the stimulated contraction of the third axillary muscle was graded as a function of stimulus frequency from $40 \mathrm{~Hz}$ to $90 \mathrm{~Hz}$. Based on this finding, Li, $\mathrm{Wu}^{27}$ designed a feedback PD controller for horizontal flight control via the graded outputs from the third axillary muscles (Figure 6). By collecting the displacement, velocity, and direction of a flying beetle as the inputs, the controller commanded the third axillary muscles with modulated pulses. When the deviation exceeded the control limit, the simultaneous stimulation of the third axillary muscle and the basalar muscle was triggered because this combined stimulation would induce a larger turning effect. Even though it was still limited by our knowledge of insect flight mechanisms, the controller achieved feedback flight control and over $70 \%$ of flights had a deviation of less than $1 \mathrm{~m}$. 
a
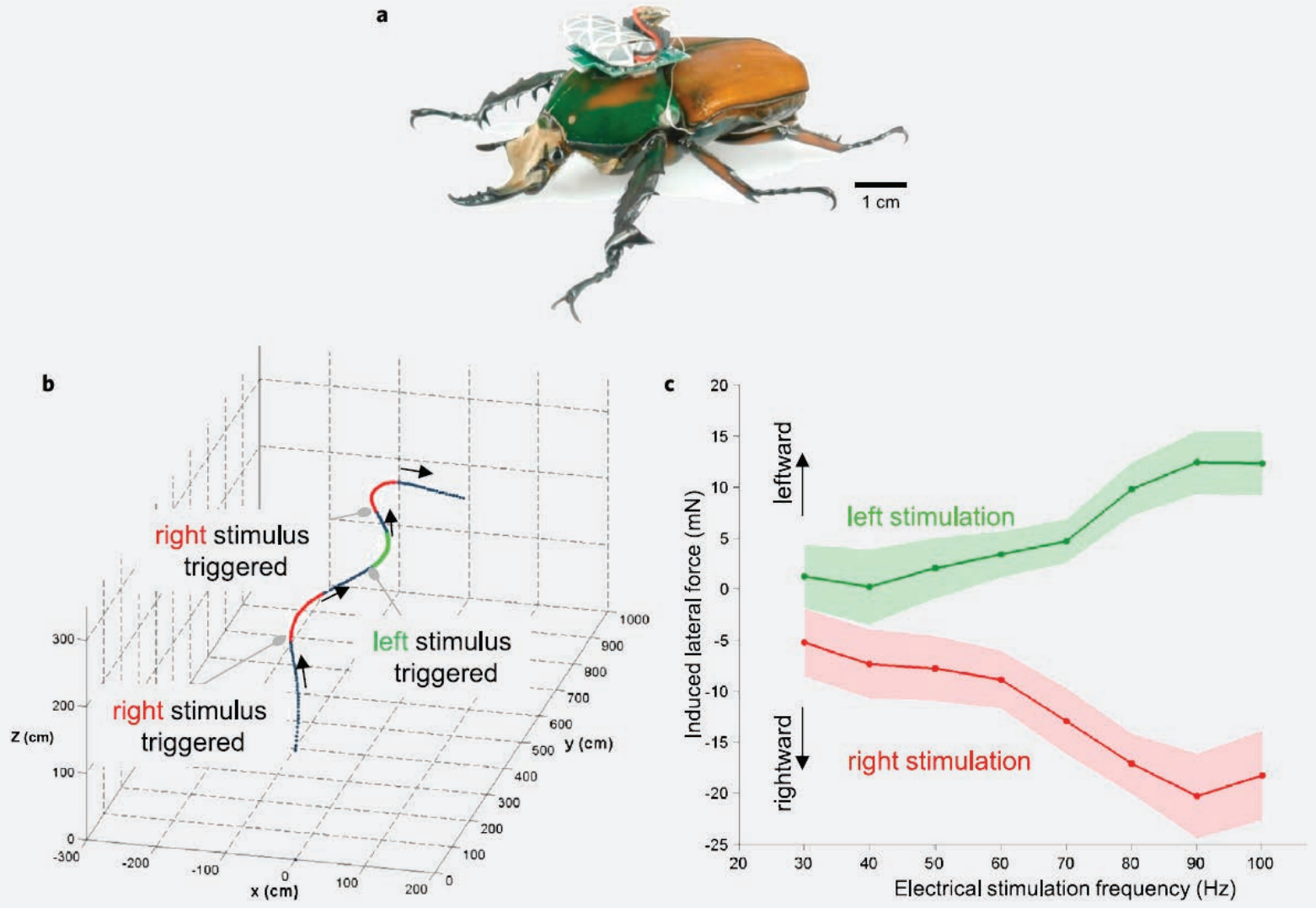

Figure 5. Flight directional control via stimulation of the third axillary ( $3 A x)$ muscles. (a) A live beetle with a miniature wireless backpack. The wires from the backpack were implanted into the left and right $3 A x$ muscles (working electrodes) and the mesothorax center hemolymph (counter electrode). (b) Electrical stimulation in sequence of the left and right $3 A x$ muscles produced left and right turns, respectively, resulting in a zigzag flight path. The black trajectory segments indicate periods of no stimulation. The red and green trajectory segments indicate right and left stimulation periods, respectively. (c) Electrical stimulation of the $3 A x$ muscle induced graded lateral forces in flight as a function of stimulus frequency. The green and red lines show the average lateral forces for left and right stimulations, respectively. A positive value denotes a leftward force and a negative value denotes a rightward force. The shaded areas denote the $95 \%$ confidence interval.

\section{Other body parts}

Apart from the wings, some other body parts, like the legs and abdomen, are involved in flight control as well ${ }^{40,41,49,50}$. Even though they cannot steer the flight as well as the wings, they contribute to yaw correction and balancing perturbations. Specifically, head rotations have assisted in correctional flight maneuvers and orienting the body to the head ${ }^{51,52}$. Abdominal deformations have been widely observed in the flight of bees, flies, and locusts ${ }^{39,41,53}$. Movements of the hindleg or changes in posture have been verified in the flight control of locusts, crickets, and bees ${ }^{49,50,54}$. Inspired by these studies, body movements have been used in the design of hybrid robots.

Bozkurt, Gilmour $\mathrm{Jr}^{22}$ stimulated the neck muscle of a flying moth with a wireless stimulation probe during a balloon-assisted flight. The $20-\mathrm{Hz}$ stimulation of the neck muscle elicited a rotation of the head of the moth. A flying insect moves its eyes by moving its head to stabilize the retinal image of the target and the head rotations precede the contractions of the wing muscles in flight turning ${ }^{55}$. Thus, controlled yawing of the moth was induced by stimulating the neck muscle.

Since the moth's abdomen plays an important role in flight stabilization, flight control of a moth can be achieved by inducing abdominal deflections. Daly, Mercier ${ }^{28}$ attached a wireless controller with a tungsten stimulator to the abdomen of a moth. The stimulator was inserted into the central nervous system in the abdomen, which sent pulses to induce abdominal deflections $\left(\sim 10^{\circ}\right)$. The system weighed $1 \mathrm{~g}$ in total and the moth maintained its flight with the system mounted. As a consequence, in flight, the induced abdominal deflections led to turning responses. 


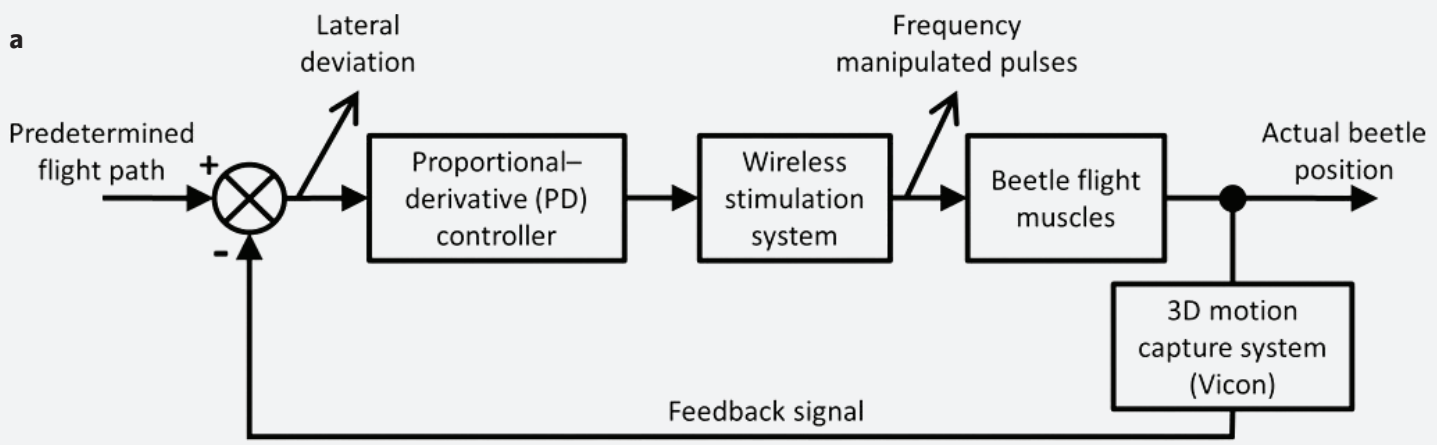

b

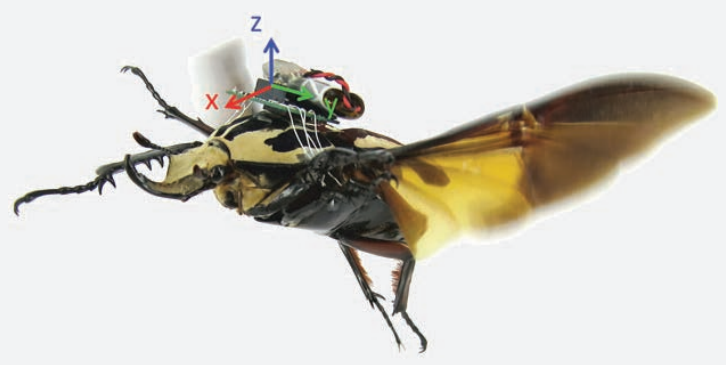

c

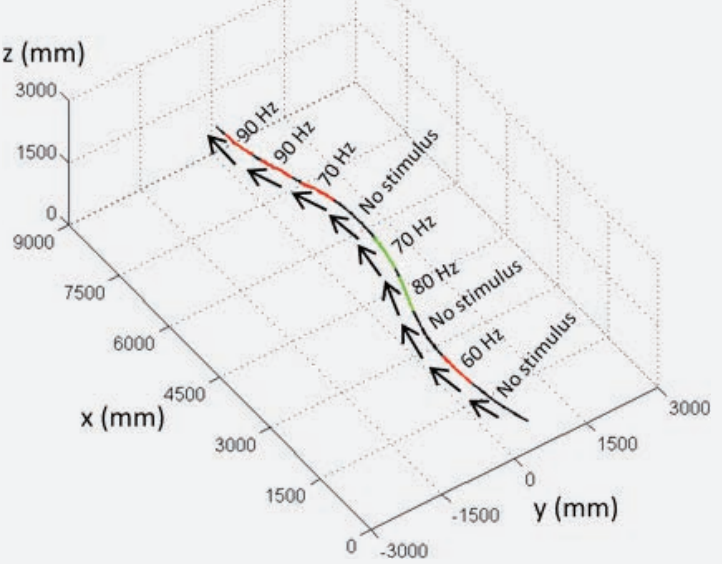

Figure 6. Feedback flight control for insect-computer hybrid flying robot. (a) Schematic of the feedback control system. The system reads the realtime position of the flying beetle as feedback information and modulates the stimulation frequencies based on the positional deviations. (b) Wireless backpack mounted on a flying beetle for muscular stimulation. In total, eight wires were soldered onto the output ports of the backpack. The terminals of the wires were inserted into four flight muscles: the basalar muscles and the third axillary muscles. (c) The flight of the beetle was modulated by the feedback control system. The output frequencies were generated according to the real-time flight status of the beetle. The segments in green and red denote the trajectories during left and right stimulation, respectively.

$\mathrm{Li}, \mathrm{CaO}^{26}$ tracked the flight postures of the forelegs of a beetle. In descriptions of flying postures, the forelegs are often mentioned as being folded or pressed closely against the body ${ }^{56,57}$. However, the beetles fully lifted and outstretched their forelegs in flight, which increases air drag. It was also observed that the forelegs swung regularly in visually induced fictive turnings. Hypothesizing that the forelegs are useful in beetle flight, the authors investigated the function of the forelegs by controlling the leg protraction muscles in free flight via a wireless backpack (Figure 1c). It was found that a beetle flew leftward when its forelegs were stimulated to swing rightward and rightward when its forelegs were stimulated to swing leftward (Figure 7). The authors concluded that the outstretching of the forelegs contributes to flight control. Thus, hybrid robots not only solve technical limitations in robotics but could be important in determining the real function of an insect muscle or structure.

\section{ONGOING AND FUTURE WORK}

With the rapid advances in interface technology and the extreme miniaturization of microcontrollers, new methodologies and equipment have been implemented in the study of insect control. Since more and more aspects of insect locomotion are being discovered, the application of insectcomputer hybrid systems is imminent. However, there are still unsolved issues. One of the outstanding issues is improving stimulation strategies. A flying insect may have as many as 13 direct flight muscles. Normally, these muscles are studied and evaluated separately from other flight muscles. However, many muscles are connected via common tendons and work together in flight ${ }^{58}$. Thus, it is necessary to explore the combined action of two or more muscles and to compare their joint effects with those of individual muscles.

The practical realization of an insect control system is another issue. There are several requirements for a con- 

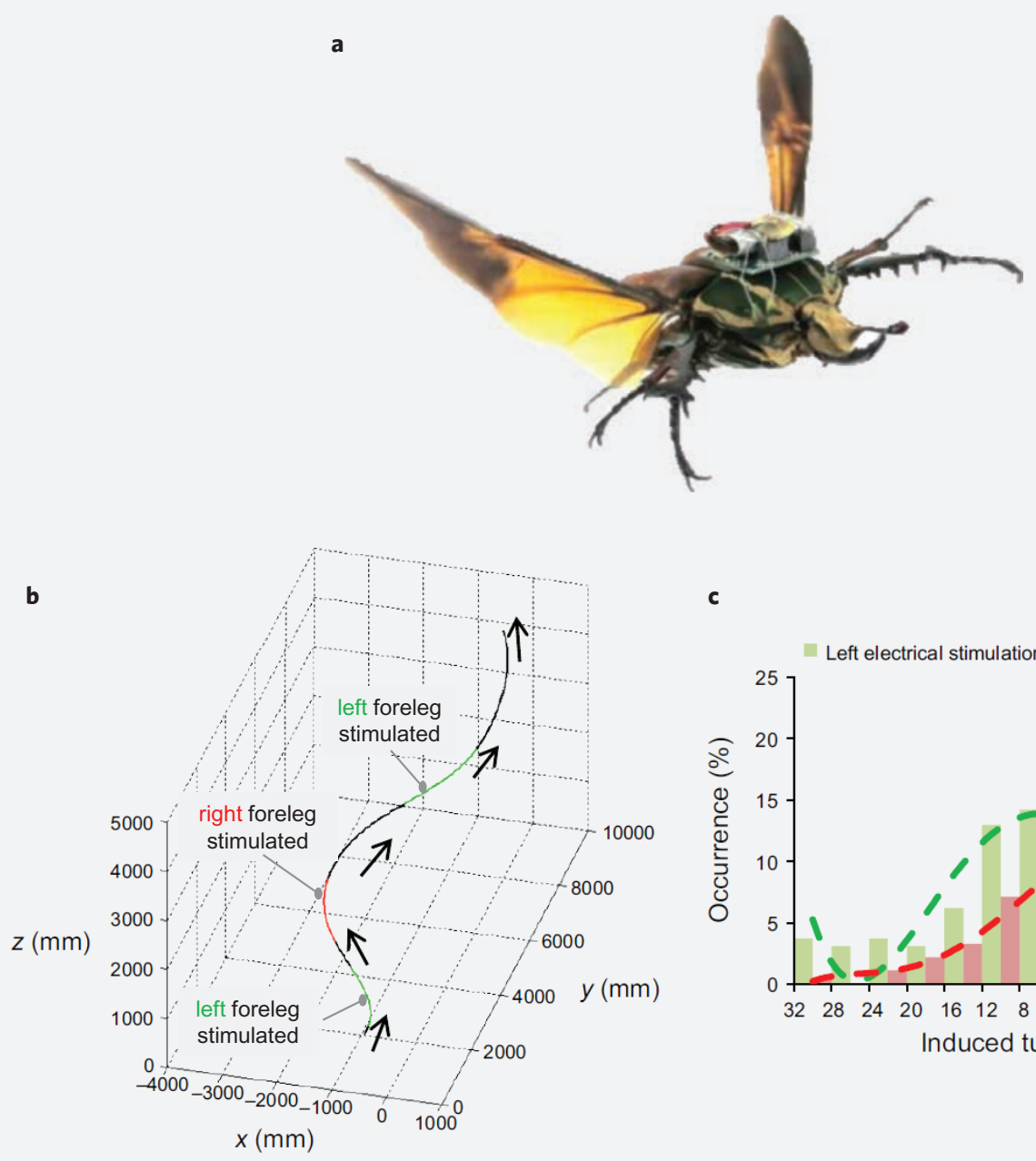

C

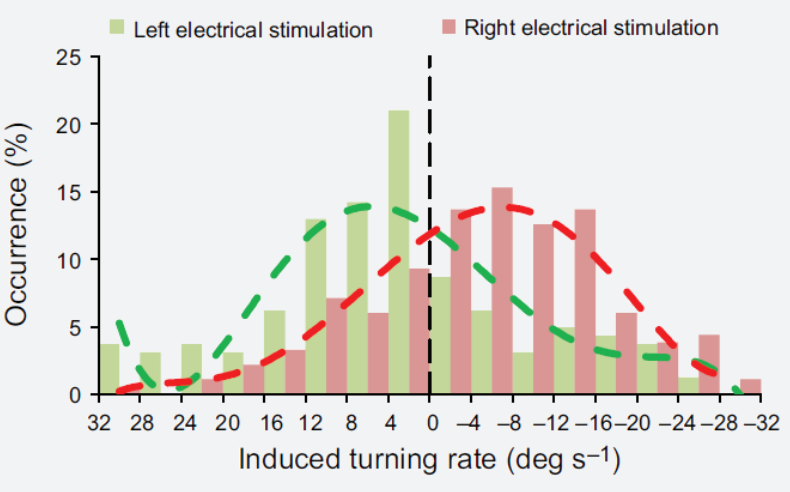

Figure 7. Flight directional control via stimulation of the foreleg protraction muscle. (a) Backpack mounted on a flying beetle to apply stimulation via two wire electrodes into each foreleg protraction muscle. (b) Electrical stimulation in sequence of the left and right protraction muscles produced left and right turns, respectively, resulting in a zigzag flight path. The black trajectory segments indicate periods of no stimulation. The red and green trajectory segments indicate right and left stimulation periods, respectively. (c) Histogram of the turning rates induced by left (green) and right (red) foreleg stimulation. The green and red dashed lines represent the polynomial fitted distribution of the turning rates induced during left and right stimulation, respectively. Positive values denote turning toward the left and negative values denote turning toward the right.

trol system for multi-purpose use. Specifically, the inherent limits to exogenous stimulation need to be evaluated systematically and an analysis of body attitudes in different movements would be helpful for behavioral identification and prediction. With improvements to the control systems, progress in following a path during walking and flying will be straightforward. Even though basic path-following has been demonstrated, the feasibility and reliability are not satisfying, as many control variables have been simplified or ignored ${ }^{15,27}$. Moreover, the cooperative swarming of insects could be realized with an improvement of the control systems.
The energy source is an important limit to practicability. Currently, hybrid robots use external batteries, which need to be charged or replaced frequently and thus, are not suitable for long-term use. There has been preliminary research on insect-mountable bio-fuel cells using cockroaches. A fuel cell could use the glucose in insect body fluids as a fuel to generate electricity ${ }^{59}$. In the future, bio-fuel cells may replace the batteries or be used to charge the batteries, which would make hybrid robots self-powered.

An insect-computer hybrid robot with controlled locomotion and able to collect data with on-board sensors would have many potential applications. In engineering, a hybrid 
robot could serve as an adaptable moving platform to carry tiny devices to locations that are difficult to reach or harmful to humans or artificial robots. Search and rescue and environmental monitoring would possibly benefit from this research. Furthermore, electrically controlled insect movement could provide suitable exemplars for the development of insect-mimicking actuators or platforms.

In biology, insect-computer hybrid robots would be useful for ethology studies, such as on the communication, collaboration, mating, and foraging of insects. Moreover, in neuroscience, insect platforms would be suitable for measuring electromyograms via specific sensors. Furthermore, the electromyogram signals could be synchronized with the stimulated responses, which are normally recorded by a real-time motion capture system. This is a commonly used methodology in kinesiology, as it demonstrates the relation between muscle activity and insect locomotion.

\section{ACKNOWLEGEMENT}

This work was supported by Singapore Ministry of Education (RG143/16, RG41/15, M0E2017-T2-2-067).

\section{REFERENCES}

1. Tipton, V.J. Insects: A success story. Am. Biol. Teach. 38(4), 205-207 (1976).

2. Sato, H. \& Maharbiz, M.M. Recent developments in the remote radio control of insect flight. Front. Neurosci. 4, 199 (2010).

3. Zhu, Y. et al. A study of arbitrary gait pattern generation for turning of a bio-inspired hexapod robot. Rob. Auton. Syst. 97, 125-135 (2017).

4. Gart, S.W. et al. Dynamic traversal of large gaps by insects and legged robots reveals a template. Bioinspir. Biomim. 13(2), 026006 (2018).

5. Minati, L. et al. Versatile locomotion control of a hexapod robot using a hierarchical network of nonlinear oscillator circuits. IEEE Access 6, 8042-8065 (2018).

6. Ma, K.Y. et al. Controlled flight of a biologically inspired, insect-scale robot. Science 340(6132), 603-607 (2013).

7. Chirarattananon, P. et al. Dynamics and flight control of a flapping-wing robotic insect in the presence of wind gusts. Interface Focus 7(1), 20160080 (2017).

8. Malka, R. et al. Principles of microscale flexure hinge design for enhanced endurance. In 2014 IEEE/RSJ International Conference on Intelligent Robots and Systems (IROS 2014), 14-18 September 2014, Chicago, USA (2014), pp. 2879-2885.

9. Wood, R.J. The first takeoff of a biologically inspired atscale robotic insect. IEEE Trans. Rob. 24(2), 341-347 (2008).
10. Li, C. et al. Cockroach-inspired winged robot reveals principles of ground-based dynamic self-righting. In 2016 IEEE/RSJ International Conference on Intelligent Robots and Systems, 9-14 October 2016, Daejeon, South Korea (2016), pp. 2128-2134.

11. Deng, S.H. et al. Experimental investigation on the aerodynamics of a bio-inspired flexible flapping wing micro air vehicle. Int. J. Micro Air Vehicles 6(2), 105-115 (2014).

12. Kutsch, W. et al. Wireless transmission of muscle potentials during free flight of a locus. J. Exp. Biol. 185(1), 367-373 (1993).

13. Holzer, R. et al. Locomotion control of a bio-robotic system via electric stimulation. In IROS'97 - Proceedings of the 1997 IEEE/RSJ International Conference on Intelligent Robot and Systems: Innovative Robotics for Real-World Applications, 11 September 1997, Grenoble, France (1996), Vols. 1-3, pp. 1514-1519.

14. Fischer, H., Kautz, H. \& Kutsch, W. A radiotelemetric 2-channel unit for transmission of muscle potentials during free flight of the desert locust, Schistocerca gregaria. J. Neurosci. Methods 64(1), 39-45 (1996).

15. Li, G. \& Zhang, D. Brain-computer interface controlled cyborg: Establishing a functional information transfer pathway from human brain to cockroach brain. PLOS One 11(3), e0150667 (2016).

16. Sanchez, C.J. et al. Locomotion control of hybrid cockroach robots. J. R. Soc. Interface 12, 20141363 (2015), http://dx.doi.org/10.1098/rsif.2014.1363.

17. Latif, T. et al. Sound localization sensors for search and rescue biobots. IEEE Sens. J. 16(10), 3444-3453 (2016).

18. Cao, F. et al. Insect-computer hybrid legged robot with user-adjustable speed, step length and walking gait. J. R. Soc. Interface 13(116), pii: 20160060 (2016), doi: 10.1098/rsif.2016.0060.

19. Vo Doan, T.T. et al. An ultralightweight and living legged robot. Soft Rob. 5(1), 17-23 (2018).

20. Cao, F. \& Sato, H. Remote radio controlled insectcomputer hybrid legged robot. In 2017 19th International Conference on Solid-State Sensors, Actuators and Microsystems (TRANSDUCERS), 16-22 June 2017, Taiwan (2017), pp. 59-62.

21. Sato, H. et al. Remote radio control of insect flight. Front. Integr. Neurosci. 3, 24 (2009).

22. Bozkurt, A., Gilmour Jr., R.F. \& Lal, A. Balloon-assisted flight of radio-controlled insect biobots. IEEE Trans. Biomed. Eng. 56(9), 2304-2307 (2009).

23. Visvanathan, K. et al. Flight initiation and directional control of beetles by microthermal stimulation. In SolidState Sensors, Actuators and Microsystems Workshop (2008). 
24. Hinterwirth, A.J. et al. Wireless stimulation of antennal muscles in freely flying hawkmoths leads to flight path changes. PLoS One 7(12), e52725 (2012).

25. Sato, H. et al. Deciphering the role of a coleopteran steering muscle via free flight stimulation. Curr. Biol. 25(6), 798-803 (2015).

26. Li, Y. et al. Role of outstretched forelegs of flying beetles revealed and demonstrated by remote leg stimulation in free flight. J. Exp. Biol. 220(19), 3499-3507 (2017).

27. Li, Y., Wu, J.B. \& Sato, H. Feedback control-based navigation of a flying insect-machine hybrid robot. Soft Rob., May 3, doi: 10.1089/soro.2017.0118 (2018) [Epub ahead of print].

28. Daly, D.C. et al. A pulsed UWB receiver SoC for insect motion control. IEEE J. Solid-State Circuits 45(1), 153-166 (2010).

29. Choo, H.Y. et al. Electrical stimulation of coleopteran muscle for initiating flight. PLoS One 11(4), e0151808 (2016).

30. Vo Doan, T.T. et al. Cyborg beetle: Thrust control of free flying beetle via a miniature wireless neuromuscular stimulator. In 28th IEEE International Conference on Micro Electro Mechanical Systems (MEMS), 18-22 January 2015, Portugal (2015).

31. Erickson, J.C. et al. Effective stimulus parameters for directed locomotion in Madagascar hissing cockroach biobot. PLoS One 10(8), e0134348 (2015).

32. Yang, Z.L. et al. A preliminary study of motion control patterns for biorobotic spiders. In 11th IEEE International Conference on Control and Automation, 18-20 June 2014, Taiwan (2014), pp. 128-132.

33. Bozkurt, A. et al. Insect-machine interface based neurocybernetics. IEEE Trans. Biomed. Eng. 56(6), 1727-1733 (2009).

34. Sato, H. et al. A cyborg beetle: Insect flight control through an implantable, tetherless microsystem. In Proceedings of the IEEE 21st International Conference on Micro Electro Mechanical Systems, 2008 (MEMS 2008), 13-17 January, Arizona, USA (2008), pp. 164-167.

35. Chung, A.J. et al. Implantable microfluidic and electronic systems for insect flight manipulation. Microfluid. Nanofluid. 13(2), 345-352 (2012).

36. Beatus, T., Guckenheimer, J.M. \& Cohen, I. Controlling roll perturbations in fruit flies. J. R. Soc. Interface 12(105), pii: 20150075 (2015), doi: 10.1098/rsif.2015.0075.

37. Ando, N., Shimoyama, I. \& Kanzaki, R. A dual-channel FM transmitter for acquisition of flight muscle activities from the freely flying hawkmoth, Agriusconvolvuli. J. Neurosci. Methods 115(2), 181-187 (2002).

38. Mohseni, P. et al. An ultralight biotelemetry backpack for recording EMG signals in moths. IEEE Trans. Biomed. Eng. 48(6), 734-737 (2001).
39. Zanker, J.M. How does lateral abdomen deflection contribute to flight control of Drosophila melanogaster? J. Comp. Physiol. A 162(5), 581-588 (1988).

40. Baader, A. The posture of the abdomen during locust flight: Regulation by steering and ventilatory interneurones. J. Exp. Biol. 151(1), 109-131 (1990).

41. Luu, T. et al. Honeybee flight: A novel 'streamlining' response. J. Exp. Biol. 214(13), 2215-2225 (2011).

42. Whitmire, E. et al. Kinect-based system for automated control of terrestrial insect biobots. Conf. Proc. IEEE Eng. Med. Biol. Soc. 2013, 1470-1473 (2013), doi: 10.1109/ EMBC.2013.6609789.

43. Cao, F. et al. A biological micro actuator: Graded and closed-loop control of insect leg motion by electrical stimulation of muscles. PLOS One 9(8), e105389 (2014).

44. Li, Y. et al. Controlled banked turns in coleopteran flight measured by a miniature wireless inertial measurement unit. Bioinspir. Biomim. 11(5), 056018 (2016).

45. Zhang, C. et al. Fuzzy-controlled living insect legged actuator. Sens. Actuators A Phys. 242, 182-194 (2016).

46. Van Truong, T. et al. Flight behavior of the rhinoceros beetle Trypoxylus dichotomus during electrical nerve stimulation. Bioinspir. Biomim. 7(3), 036021 (2012).

47. Chapman, R.F., Simpson, S.J. \& Douglas, A.E. The Insects: Structure and Function (Cambridge University Press, UK, 2013).

48. Goldsworthy, G.J. \& Wheeler, C.H. Insect Flight (CRC Press, 1989).

49. Arbas, E.A. Control of hindlimb posture by wind-sensitive hairs and antennae during locust flight. J. Comp. Physiol. A 159(6), 849-857 (1986).

50. May, M.L. \& Hoy, R.R. Leg-induced steering in flying crickets. J. Exp. Biol. 151, 485-488 (1990).

51. Robert, D. \& Rowell, C. Locust flight steering. I. Head movements and the organization of correctional manoeuvres. J. Comp. Physiol. A Sens. Neural Behav. Physiol. 171(1), 41-52 (1992).

52. Hensler, K. \& Robert, D. Compensatory head rolling during corrective flight steering in locusts. J. Comp. Physiol. A Neuroethol. Sens. Neural Behav. Physiol. 166(5), 685693 (1990).

53. Camhi, J.M. Sensory control of abdomen posture in flying locusts. J. Exp. Biol. 52(3), 533 (1970).

54. Combes, S.A. \& Dudley, R. Turbulence-driven instabilities limit insect flight performance. Proc. Natl. Acad. Sci. U.S.A. 106(22), 9105-9108 (2009).

55. Strausfeld, N.J. The head-neck system of the blowfly Calliphora: 1. Anatomic organization of neck muscles, motoneurons, and multimodal and visual inputs. In The 
Head-Neck Sensory Motor System (Oxford University Press, 2012).

56. Nachtigall, W. Insects in Flight: A Glimpse Behind the Scenes in Biophysical Research (McGraw-Hill Companies, 1974).

57. Borst, A. Time course of the houseflies' landing response. Biol. Cybern. 54(6), 379-383 (1986).
58. Dickinson, M.H. \& Tu, M.S. The function of dipteran flight muscle. Comp. Biochem. Physiol Part A Physiol. 116(3), 223-238 (1997).

59. Shoji, K. et al. Biofuel cell backpacked insect and its application to wireless sensing. Biosens. Bioelectron. 78, 390-395 (2016). 